\title{
How to integrate monoclonal antibodies targeting the calcitonin gene-related peptide or its receptor in daily clinical practice
}

\author{
Cindy Tiseo, Raffaele Ornello, Francesca Pistoia and Simona Sacco*
}

\begin{abstract}
Background: Migraine is a major public health issue associated with significant morbidity, considerable negative impact on quality of life, and significant socioeconomic burden. Preventive treatments are required to reduce the occurrence and the severity of acute attacks and to minimize the use of abortive medications and the associate risk of drug-related adverse events, as well as the onset of medication-overuse headache and chronification of migraine. We performed a review of all available evidence on the safety and efficacy of monoclonal antibodies targeting the calcitonin gene-related peptide or its receptor for the preventive treatment of migraine to provide evidence-based guidance on their use in clinical practice.

Abstract main body: Monoclonal antibodies targeting the calcitonin gene-related peptide or its receptor are mechanism-specific drugs for the preventive treatment of migraine. Double-blind randomized clinical trials have shown that monoclonal antibodies targeting the calcitonin gene-related peptide or its receptor are effective across all the spectrum of migraine patients who require prevention and have a good safety and tolerability profile. Nevertheless, high costs limit the affordability of those drugs at the moment.

Conclusions: Specificity, long half-life, efficacy, tolerability, and ease of use make monoclonal antibodies targeting the calcitonin gene-related peptide or its receptor an appealing treatment option for migraine prevention. Optimal strategies to manage treatment over time still need to be clarified with real-life data.
\end{abstract}

Keywords: Migraine, Calcitonin gene-related peptide, Preventive treatment, Monoclonal antibodies

\section{Introduction}

Migraine is a chronic neurologic disease affecting around $15 \%$ of adult subjects, with a higher female prevalence [1-3]. It is a major public health issue associated with significant morbidity, considerable negative impact on quality of life, and considerable socioeconomic burden [4]. Patients with migraine attacks recurring at high frequency, or being associated with pain of severe intensity, or determining a reduction of quality of life require preventive treatments. In recent years, advances in the understanding of migraine pathophysiology paved the way for the development of migraine-specific preventive

\footnotetext{
* Correspondence: simona.sacco@univaq.it

Neuroscience Section, Department of Applied Clinical Sciences and Biotechnology, University of L'Aquila, 67100 L'Aquila, Italy
}

treatments. Early after the discovery of calcitonin-gene related peptide (CGRP) [5, 6], it was clear that it would have a crucial role in the pathophysiology of migraine [7-12]. CGRP is a neuropeptide existing in 2 isoforms, $\alpha$ and $\beta$. The $\alpha$ isoform, which is primarily implied in the pathogenesis of migraine pain, is a 37 -aminoacid peptide synthesized by peripheral sensory neurons and by numerous sites in the central nervous system through alternative splicing of the calcitonin gene mRNA [5]. The $\beta$ isoform is encoded from a different gene and it is expressed primarily by the enteric sensory system [8]. The receptors for members of the CGRP peptide family consist of two G protein-coupled receptors, the calcitonin-like receptor (CLR; a seven-transmembrane receptor component) and the receptor component protein $(\mathrm{RCP})$, interacting with

(c) The Author(s). 2019 Open Access This article is distributed under the terms of the Creative Commons Attribution 4.0 International License (http://creativecommons.org/licenses/by/4.0/), which permits unrestricted use, distribution, and 
the receptor activity-modifying protein 1 (RAMP1). CGRP receptor is expressed by the trigeminal neurons, the smooth muscle cells of peripheral intracranial vasculature, the dura mater, and the brainstem [8].

Experimental studies revealed that CGRP levels are increased during the migraine attack [9] and tend to normalize together with pain relief [10]. Interestingly, the intravenous administration of CGRP can induce migraine-like headache in migraineurs but not in healthy subjects [13]. The current hypothesis on migraine pathogenesis suggests that migraine initiates in the brain with cortical and subcortical changes inducing the activation of the trigeminovascular system with subsequent transmission of pain signals to the thalamus [14]. The activation of trigeminovascular system seems a crucial step for the complete expression of migraine attack and its accompanying symptoms [15]. Following the activation of the trigeminovascular system, CGRP is released at trigeminal endings and induces vasodilation of the intracranial arteries, modulates neuronal excitability through the facilitation of pain transmission, and activates neurogenic inflammation.

Based on all those findings, CGRP gained importance as a potential pharmacological target for migraine prevention. Small molecules acting as competitive CGRP receptor antagonists, the gepants, have been proven effective in the acute and prophylactic treatment of migraine attacks, but their clinical development was limited by safety concerns regarding liver toxicity following continuous exposure to the initial drugs [16]. Therefore, the attention shifted to the development of monoclonal antibodies (MoAbs) targeting CGRP or its receptor. The mechanism of action of MoAbs in migraine treatment includes the modulation of CGRP-induced pain transmission and the reduction of both peripheral and central sensitization through the removal of the excess of released CGRP (CGRP MoAbs) or the block of the ligand from binding the CGRP receptor (CGRP receptor MoAbs) [17].

We performed a review of all available evidence on the safety and efficacy of CGRP(r) MoAbs in patients with migraine to provide evidence-based guidance on their use in clinical practice.

\section{Methods}

We searched papers indexed in PubMed and Scopus from inception to December 2018 using the following search terms: "migraine OR headache AND (CGRP OR eptinezumab OR galcanezumab OR fremanezumab OR erenumab)". We also performed a manual search among contributions presented to the main headache conferences including the European Headache Federation, the International Headache Conference, and the American Academy of Neurology during the years 2017 and 2018. A manual search among references lists and Google Scholar citations of selected articles and reviews was also performed. Only studies published in English were considered.

We included data from phase III clinical trials, or phase II trials if phase III trials were lacking, which tested the CGRP(r) MoAbs doses to be used in clinical practice.

\section{Available evidence on efficacy}

To date, four IgG MoAbs acting on the CGRP pathway have been developed and tested in humans: eptinezumab, erenumab, fremanezumab and galcanezumab [18-31]. Erenumab is a fully human MoAb, binding the CGRP receptor, while eptinezumab, fremanezumab, and galcanezumab are fully humanized MoAbs binding CGRP.

Eptinezumab has been studied in a phase II randomized clinical trial (RCT) [18] for the prevention of episodic migraine (EM) at the dose of $1000 \mathrm{mg}$. Ongoing phase III RCTs are testing the quarterly intravenous administration of eptinezumab $30 \mathrm{mg}, 100 \mathrm{mg}$ and $300 \mathrm{mg}$ for the prevention of frequent EM (PROMISE-1) [19-21], and of eptinezumab $100 \mathrm{mg}$ and $300 \mathrm{mg}$ for chronic migraine (CM) (PROMISE-2) [22, 23]. Erenumab has been studied in phase III RCTs (STRIVE and ARISE) for the prevention of EM [24, 25] and in phase II RCT [26] for the prevention of CM at the doses of $70 \mathrm{mg}$ and $140 \mathrm{mg}$ administered subcutaneously monthly. Fremanezumab has been investigated in phase III RCTs at the doses of $225 \mathrm{mg}$ administered monthly and $675 \mathrm{mg}$ quarterly for the prevention of EM (HALO-EM) [27], and at the doses of $675 \mathrm{mg}$ quarterly and of $225 \mathrm{mg}$ monthly (with a single loading dose of $675 \mathrm{mg}$ ) for the prevention of CM (HALO-CM) [28]. Galcanezumab has been studied in phase III RCTs at the doses of $120 \mathrm{mg}$ (with a single loading dose of $240 \mathrm{mg}$ ) and of $240 \mathrm{mg}$ administered monthly for the prevention of EM (EVOLVE-1 and EVOLVE-2) [29, 30] and CM (REGAIN) [31].

Figures 1 and 2 provide an overview of the results on the main efficacy endpoints of CGRP(r) MoAbs observed in RCTs. In patients with EM (Fig. 1), erenumab, fremanezumab, and galcanezumab determined a modest but significant decrease, ranging from 1 to 2 days, in the mean number of MMD, a significant reduction in the monthly acute medication use and a meaningful improvement in function with respect to placebo after 12-24 weeks of treatment. Treatment with eptinezumab was superior to placebo in the reduction of the number of MMD at weeks $5-8$, but a claimed effect was not confirmed at week 12; besides, eptinezumab $1000 \mathrm{mg}$ determined a reduction of monthly acute medication use, but a non-significant improvement in function compared with placebo [18]. Nevertheless, preliminary results of the phase III PROMISE-1 RCT [19] showed that treatment with eptinezumab determined a significant reduction of MMD and a higher proportion of patients achieving $\geq 50 \%$ 
Outcome: change in MMDs from baseline to month 3-6 Active vs Placebo

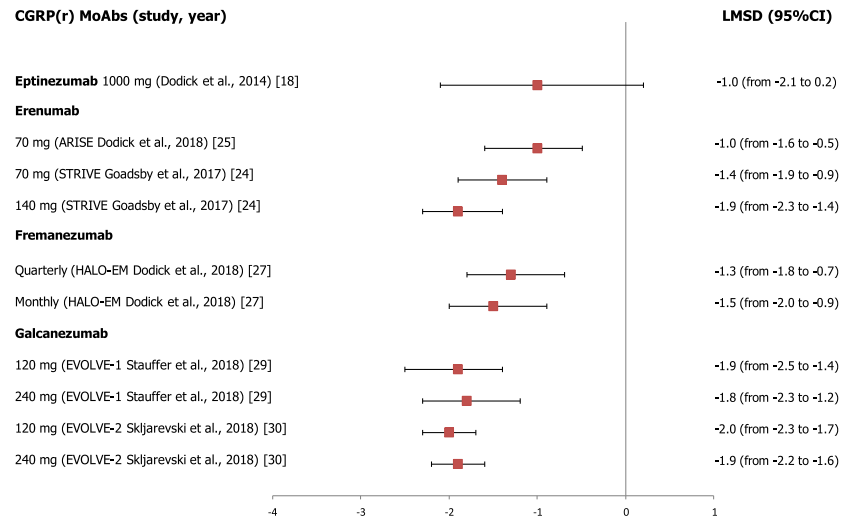

Outcome: $\geq 50 \%$ reduction in MMDs from baseline to month 3-6 Active vs Placebo

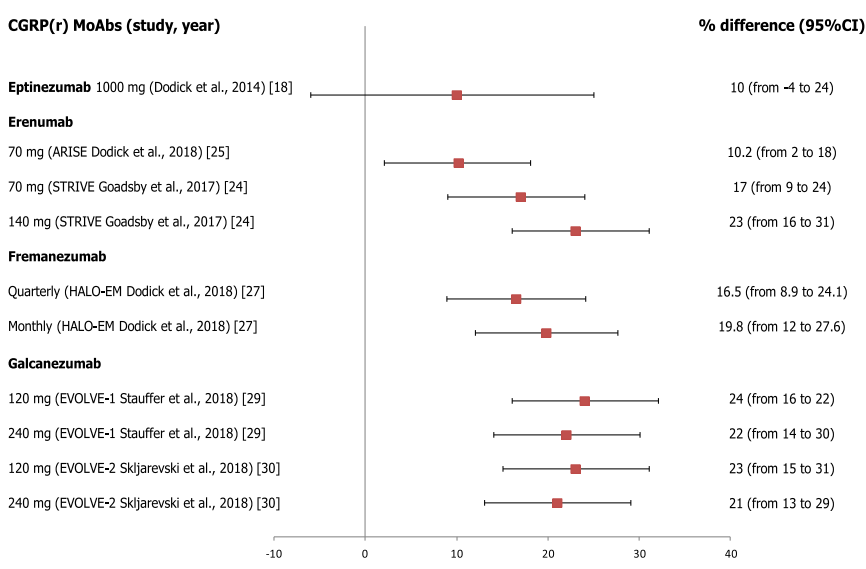

Outcome: change in MMDs with acute medication use from baseline to month 3-6 Active vs Placebo

\begin{tabular}{|c|c|c|}
\hline CGRP(r) MoAbs (study, year) & & LMSD (95\%CI) \\
\hline \multicolumn{3}{|l|}{ Erenumab } \\
\hline $70 \mathrm{mg}$ (ARISE Dodick et al., 2018) [25] & $\longmapsto$ & $-0.6($ from -1.0 to -0.2$)$ \\
\hline $70 \mathrm{mg}$ (STRIVE Goadsby et al., 2017) [24] & •— & $-0.9($ from -1.2 to -0.6$)$ \\
\hline 140 mg (STRIVE Goadsby et al., 2017) [24] & ロロ & -1.4 (from -1.7 to -1.1 ) \\
\hline \multicolumn{3}{|l|}{ Fremanezumab } \\
\hline Quarterly (HALO-EM Dodick et al., 2018) [27] & $\longmapsto$ & -1.3 (from -1.8 to -0.8 ) \\
\hline Monthly (HALO-EM Dodick et al., 2018) [27] & $\longmapsto$ & $-1.4($ from -1.8 to -0.9 ) \\
\hline \multicolumn{3}{|l|}{ Galcanezumab } \\
\hline $120 \mathrm{mg}$ (EVOLVE-1 Stauffer et al., 2018) [29] & $\longmapsto$ & -1.8 (from -2.3 to -1.3 ) \\
\hline 240 mg (EVOLVE-1 Stauffer et al., 2018) [29] & $\longmapsto$ & $-1.6($ from -2.1 to -1.1$)$ \\
\hline $120 \mathrm{mg}$ (EVOLVE-2 Skljarevski et al., 2018) [30] & $\longmapsto$ & -1.8 (from -2.4 to -1.2 ) \\
\hline $240 \mathrm{mg}$ (EVOLVE-2 Skljarevski et al., 2018) [30] & $\longmapsto$ & -1.7 (from -2.4 to -1.2 ) \\
\hline
\end{tabular}

Fig. 1 Data showing the results of treatment with CGRP(r) MoAbs on the main efficacy endpoints in patients with episodic migraine 
Outcome: change in MMDs from baseline to month 3 Active vs Placebo

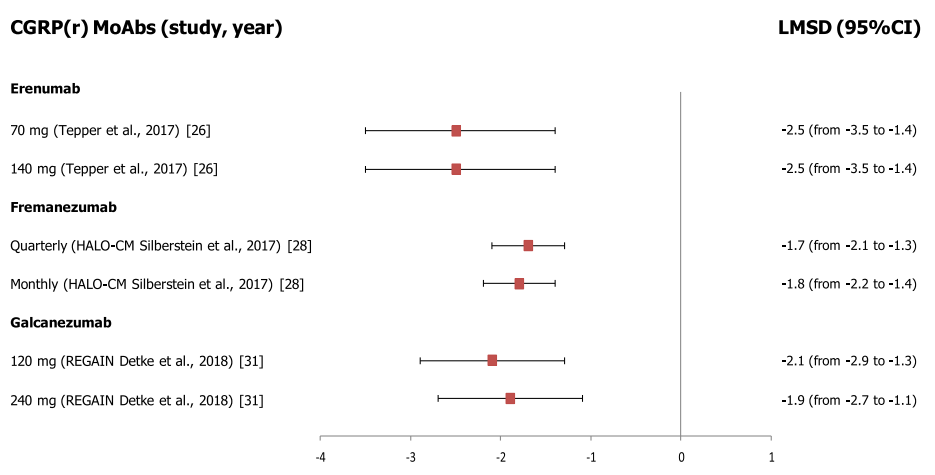

Outcome: $\mathbf{2 5 0} \%$ reduction in MMDs from baseline to month 3 Active vs Placebo

CGRP(r) MoAbs (study, year)

Erenumab

$70 \mathrm{mg}$ (Tepper et al., 2017) [26]

$140 \mathrm{mg}$ (Tepper et al., 2017) [26]

Fremanezumab

Quarterly (HALO-CM Silberstein et al., 2017) [28]

Monthly (HALO-CM Silberstein et al., 2017) [28]

Galcanezumab

$120 \mathrm{mg}$ (REGAIN Detke et al., 2018) [31]

$240 \mathrm{mg}$ (REGAIN Detke et al., 2018) [31]

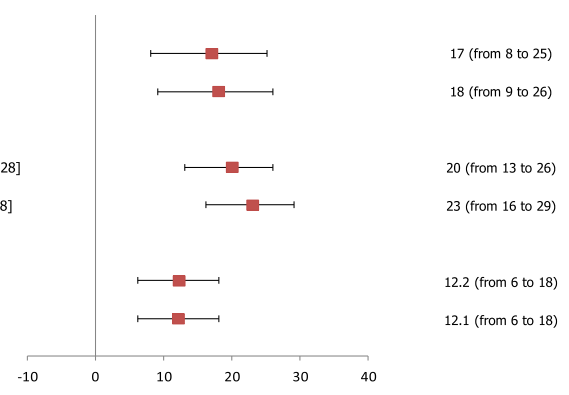

Outcome: change in MMDs with acute medication use from baseline to month 3

Active vs Placebo

CGRP(r) MoAbs (study, year)

LMSD (95\%CI)

Erenumab

$70 \mathrm{mg}$ (Tepper et al., 2017) [26]

$140 \mathrm{mg}$ (Tepper et al., 2017) [26]

Fremanezumab

Quarterly (HALO-CM Silberstein et al., 2017) [28]

Monthly (HALO-CM Silberstein et al., 2017) [28]

Galcanezumab

$120 \mathrm{mg}$ (REGAIN Detke et al., 2018) [31]

240 mg (REGAIN Detke et al., 2018) [31]
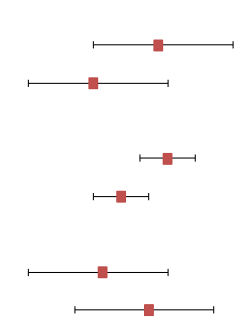

om -3.3 to -1.8

$-2.0($ from -2.8 to -1.3$)$

2.6 (from -3.3 to -1.8 )

-1.8 (from -2.1 to -1.5 )

-2.3 (from -2.6 to -2.0 )

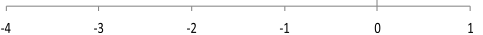

Fig. 2 Data showing the results of CGRP(r) MoAbs on the main efficacy endpoints in patients with chronic migraine 
Table 1 Recommended dose regimens of CGRP MoAbs for migraine prevention

\begin{tabular}{lllll}
\hline Drug & Route of administration & Dosage forms & Loading dose & $\begin{array}{l}\text { Recommended } \\
\text { dose regimen }\end{array}$ \\
\hline Erenumab & sc injection & $70 \mathrm{mg} / \mathrm{mL}$ solution & Not required & $70 \mathrm{mg}$ monthly \\
Fremanezumab & sc injection & $225 \mathrm{mg} / 1.5 \mathrm{~mL}$ solution & Not required & $225 \mathrm{mg}$ monthly \\
& & & Not required & $675 \mathrm{mg}$ quarterly \\
Galcanezumab & sc injection & $120 \mathrm{mg} / \mathrm{mL}$ solution & $240 \mathrm{mg}^{\mathrm{c}}$ & $120 \mathrm{mg}$ monthly \\
\hline
\end{tabular}

${ }^{\mathrm{a}}$ Two consecutive injections of $70 \mathrm{mg}$ each may be reasonable for selected patients (see text)

${ }^{\mathrm{b}}$ Three consecutive injections of $225 \mathrm{mg}$ each

'Two consecutive injections of $120 \mathrm{mg}$ each

reduction of MMD with respect to placebo. A further clinically meaningful finding reported by RCTs is the evidence of a higher significant proportion of patients that at weeks 12-24 achieved at least 50\% reduction of MMD with CGRP(r) MoAbs (from $40 \%$ to $62 \%$ ) than with placebo (from $27 \%$ to $39 \%$ ) $[18,24,25,27,29,30]$. Notably, a proportion of patients with EM treated with CGRP(r) MoAbs had a complete response, i.e. no migraine episodes; at week 12 the proportion of complete responses was $16 \%$ for eptinezumab [18] and 35.5\% for galcanezumab $120 \mathrm{mg}$ [32], while at week 24 the proportion ranged from $11.5 \%$ to $15.6 \%$ for galcanezumab $120 \mathrm{mg}$, and from $13.8 \%$ to $14.6 \%$ for galcanezumab $240 \mathrm{mg}[29,30]$.

In patients with CM (Fig. 2), a 12-week treatment with erenumab, fremanezumab, and galcanezumab was associated with a significant decrease, ranging from 2 to 3 days, in the mean number of MMD [24, 26, 29], a higher rate of patients with at least $50 \%$ reduction of MMD [26, 28, 31], a significant reduction, ranging from 2 to 3 days, in the monthly acute medication use [26, 28, 31], and a meaningful improvement in function compared with placebo $[28,31]$. Preliminary results of the phase III PROMISE-2 RCT $[22,23]$ showed that treatment with eptinezumab was associated with a significant reduction of MMD (mean MMD reduction: eptinezumab $100 \mathrm{mg}$, - 7.7; eptinezumab $300 \mathrm{mg},-8.2$; placebo, -5.6) and with a higher proportion of patients achieving at least $50 \%$ reduction in MMDs (eptinezumab $100 \mathrm{mg}$, 57.6\%; eptinezumab $300 \mathrm{mg}$, 61.4\%; placebo, 39.3\%) compared with placebo from baseline to week 12 .
CGRP(r) MoAbs have shown superiority even in reducing the burden of non-head-pain symptoms of migraine, including nausea or vomiting, photophobia, and phonophobia. Indeed, post-hoc analyses of the phase II [33], of the HALO-EM [34] and HALO-CM trials [35] on fremanezumab, and of the EVOLVE-1, EVOLVE-2 and REGAIN RCTs [36] on galcanezumab showed that patients in the treatment group yielded a modest but significantly greater reduction in the mean number of days with nausea or vomiting and with phonophobia and photophobia compared with placebo from baseline to week 12 in both EM and CM patients. Moreover, evidence from preliminary data on galcanezumab suggest the possible benefit of CGRP(r) MoAbs on reducing MMD with prodromal symptoms in both patients with EM and CM [36].

Overall, data on the efficacy of CGRP(r) MoAbs on migraine associated symptoms seem to suggest that their effect goes beyond the mere control of the pain and that is possibly implicated in preventing the activation of those mechanisms leading to the complete clinical manifestation of the migraine attack. Less clear is the role of CGRP(r) MoAbs on aura symptoms; preliminary data on galcanezumab have shown the superiority of treatment compared with placebo in reducing MMD with aura in EM, but not in CM patients [36]. It would be interesting to understand whether CGRP(r) MoAbs, which cross the intact blood-brain barrier only in small amounts, might indirectly inhibit cortical spreading depression [37].

Table 2 Practical issues about CGRP(r) MoAbs versus other migraine preventive treatments with established efficacy

\begin{tabular}{lllll}
\hline & CGRP(r) MoAbs & Antiepileptics & B-blockers & OnabotulinumtoxinA \\
\hline $\begin{array}{l}\text { Route of } \\
\text { administration }\end{array}$ & $\begin{array}{l}\text { Intravenous or } \\
\text { subcutaneous } \\
\text { injection }\end{array}$ & Oral & Oral & Intramuscular \\
Dosing frequency & Monthly or quarterly & $\geq 1$ daily & $\geq 1$ daily & Quarterly \\
Adverse effects & Rare & Common & Infrequent & Rare \\
$\begin{array}{l}\text { Pregnancy and } \\
\text { nursing }\end{array}$ & Contraindicated & Contraindicated & Possible with caution in the first & Contraindicated \\
Costs & trimester of pregnancy Not & recommended during breastfeeding & Medium \\
\hline
\end{tabular}

CGRP(r) indicates Calcitonin gene-related peptide (receptor), MoAbs Monoclonal antibodies 


\section{Dose and route of administration}

CGRP(r) MoAbs are administered with subcutaneous or intravenous injections. Their large dimensions, the relatively low permeability through cell membranes and instability in the gastrointestinal tract make CGRP(r) MoAbs unsuitable for oral administration. All CGRP(r) MoAbs have a long half-life in blood and a corresponding long duration of action, thus allowing for long administration intervals with monthly or quarterly dose. Moreover, none of these agents requires dose titration.

Currently, erenumab, fremanezumab, and galcanezumab have been approved at the doses of $70 \mathrm{mg}, 225 \mathrm{mg}$, and $120 \mathrm{mg}$ respectively. Table 1 summarizes the recommended dose regimens for the approved CGRP(r) MoAbs. Erenumab, fremanezumab and galcanezumab are all available for self-administration with a subcutaneous single-dose prefilled pen. The suggested sites of subcutaneous injection are upper arms, lower abdomen/belly/ waistline, and front of thighs. For higher treatment dose regimen multiple consecutive injections of a single-dose might be required; the same body site can be used, but it is recommended not to inject the exact location of the first one. It would be reasonable that physicians administer the first treatment dose, to provide proper training to patients or caregivers and to evaluate possible allergic reactions.

\section{Patients eligible for treatment}

As for other preventive treatments, before prescribing an CGRP(r) MoAb preliminary considerations on migraine and patient's characteristics are mandatory. Patients to be considered for the treatment with CGRP(r) MoAbs should suffer from migraine with or without aura according to the criteria of the International Classification of Headache Disorders [38] and have at least 4 MMDs. In RCTs, CGRP(r) MoAbs have been tested in patients with EM with migraine attack frequency of at least 4 MMDs, in those with high frequency EM with at least 8 MMDs, and in those with CM with at least 15 monthly headache days, including at least 8 MMDs.

Both male and female patients aged from 18 to 70 years may benefit from treatment with CGRP(r) MoAbs for migraine prophylaxis. We have no data about the safety and efficacy of those agents in patients younger than 18 years and older than 70 years, because they were not included in RCTs. It reasonable to think that the patient's eligibility to CGRP(r) MoAbs should go beyond age restrictions and that more important is the assessment of intercurrent clinical conditions that would contraindicate their use.

No head-to-head studies comparing CGRP(r) MoAbs with other preventive treatments are available allowing comparisons in term of efficacy [39] but the tolerability profile appears much better than that of the available drugs (Table 2). This is a very relevant point considering that in migraine, preventive treatment is mainly aimed to improve quality of life and that in many cases side effects overcome benefits related to prevention of attacks. Additionally, the infrequent administration, the lack of titration, the quick onset of action, and of the absence of interactions with other drugs, make the treatment with the CGRP(r) MoAbs appealing for physicians and patients. The main limitation of CGRP(r) MoAbs is represented by high costs which cannot make those drugs the first-line treatment for migraine prevention. In the clinical setting, it is reasonable to use $\operatorname{CGRP}(r)$ MoAbs in patients who failed treatment with at least two preventive drug classes because of lack of efficacy or intolerable side effects. For patients with CM, it might be reasonable to require failure of an adequate trial with onabotulinumtoxinA before starting the CGRP MoAbs. Moreover, some patients may have contraindications to oral preventive drugs further limiting the possibilities of treatment. In patients who are overweight and obese and have comorbid depression most of the available preventive drugs are contraindicated; in this group of patients, CGRP(r) MoAbs may represent the first-line treatment together with onabotulinumtoxinA if they have CM.

\section{Screening before initiating CGRP( $r)$ MoAbs}

Initiation of CGRP(r) MoAbs should be preceded by a detailed history and a complete physical examination in order to evaluate the patient eligibility and to exclude possible contraindications to their use. Special attention should be paid to childbearing potential women since there are no adequate data on the developmental risk associated with the use of $\operatorname{CGRP}(r)$ MoAbs during pregnancy. Women of childbearing age should receive counseling on the appropriate use of methods of birth control. As a consequence of long half-life, it would be also reasonable to delay pregnancy after the last dose of CGRP(r) MoAbs. Moreover, there are no data on the presence of CGRP(r) MoAbs in human milk, therefore the effects on the breastfed infant is unknown. When evaluating to treat with $\mathrm{CGRP}(\mathrm{r}) \mathrm{MoAbs}$ women during lactation, the developmental and health benefits of breastfeeding should be considered along with the mother's clinical need for migraine specific preventive treatment.

Patients should be also screened for preexisting cardiovascular disease before initiating treatment with CGRP(r) MoAbs. Since CGRP has a potent vasodilatator effect throughout the vascular system [40], the chronic inhibition of CGRP pathway with MoAbs and has been considered dangerous because potentially able to induce hypertension and facilitate the precipitation of coronary or cerebral hypoperfusion into ischemia [41, 42]. For these reasons, patients with cardio- and cerebrovascular 
diseases including history of myocardial infarction, stroke, transient ischemic attack, unstable angina, or coronary artery bypass surgery or other revascularization procedures were excluded from available RCTs [18, 24-32]. It would be reasonable to exclude from treatment patients with comorbid cardiovascular diseases, at least as long as further studies and real world registries record the long-term effects of continuous blockade of the CGRP pathway. However, results from a placebo-controlled study in a high-risk population of patients with stable angina with a median age of 65 years, the inhibition of the CGRP receptor with erenumab did not adversely affect total exercise time in a treadmill test [43]. Moreover, erenumab showed to have no relevant effect on blood pressure and on diurnal rhythm of blood pressure in patients with migraine over a 12 weeks treatment period compared with placebo [44]. Screening for psychiatric conditions, including major depression, suicide ideation, schizophrenia and bipolar disorders, and for alcohol and drug abuse should be required. It would be reasonable to treat with caution these subgroups of patients, as they have been excluded from RCTs. Nevertheless, a subgroup analysis of phase III data in EM patients showed that compared with placebo, erenumab proved effective against migraine in patients with and without depression or anxiety history [45]. Similarly, fremanezumab demonstrated efficacy in preventive treatment of CM in patients with depressive symptoms [46].

Before initiating treatment with CGRP(r) MoAbs, no specific laboratory tests are required. MoAbs are proteins degraded by tissue into endogenous amino acids with consequent fewer safety concerns and low risk of drug-drug interaction [47]. However, it is worth considering that patients with significant laboratory abnormality, hepatic disease, abnormal liver and kidney function were excluded from RCTs [18, 24-32].

\section{Special consideration for management of CGRP( $r)$ MoAbs treatment}

Previous preventive treatment failures

CGRP(r) MoAbs have been tested in preventive treatment-naïve patients as well as in those who had an inadequate response or intolerance to one or more preventive treatment. Available studies in EM allowed the inclusion of patients with failure of up to 2 preventive drugs and studies in CM allowed the inclusion of patients with failure of up to 3 preventive drugs. Only the phase II RCT on eptinezumab in EM did not exclude patients according to the number of previous failure of preventive drugs [18]. Available evidence on erenumab $[48,49]$, fremanezumab $[50,51]$ and galcanezumab [52] suggests that CGRP(r) MoAbs are effective even in EM and CM patients with previous preventive treatment failures.
Evidences from RCTs on erenumab suggest that in patients with EM and at least 1 previous preventive failure both 70 and $140 \mathrm{mg}$ regimens are effective, while in those with at least 2 preventive failures the efficacy of erenumab $140 \mathrm{mg}$ was superior compared with placebo in reducing MMD, whereas it was only marginal with erenumab $70 \mathrm{mg}$ [48]. The phase IIIb LIBERTY trial [53] evaluating the efficacy of erenumab $140 \mathrm{mg}$ in preventing EM in patients with 2-to-4 preventive treatment failures confirmed the superiority of erenumab compared with placebo in the main efficacy endpoints [53]. Similarly, in patients with CM and previous preventive failure both doses of erenumab were effective compared with placebo in the main efficacy endpoints at month 3 and were greater in the subgroups of patients who have failed $\geq 1$ or $\geq 2$ prior preventive treatments than in patients with no prior treatment failure, with the greatest differences in patients who failed $\geq 2$ prior preventive medications and treated with erenumab $140 \mathrm{mg}$ [49]. Contrariwise, in patients with CM and at least 2 preventive failures, galcanezumab $120 \mathrm{mg}$ was more effective than galcanezumab $240 \mathrm{mg}$ in reducing MMD [52].

OnabotulinumtoxinA injections for migraine prevention were prohibited during the RCTs and for at least 4-6 months before the start of the baseline phase [26, 28, 31]. Patients who had previous use of onabotulinumtoxinA were included in RCTs but no information referring to previous efficacy of onabotulinumtoxinA and response to study treatment is available. Post-hoc analyses of phase III RCTs on galcanezumab showed that treatment significantly decreased MMD and acute migraine->specific medication use, and improved quality of life in patients who failed prior onabotulinumtoxinA treatment due to lack of efficacy or tolerability [54]. In these subgroups of patients, the greater reduction in MMD was observed with galcanezumab $240 \mathrm{mg}$ with respect to galcanezumab $120 \mathrm{mg}$, while the improvement in acute migraine-specific medication use and in quality of life was similar between the two dose regimens [54].

The results of those studies suggest that CGRP(r) MoAbs represent a valid treatment option for patients with $\mathrm{EM}$ and $\mathrm{CM}$ with previous preventive treatment failure, but also for those difficult-to-treat migraine patients in whom multiple traditional oral migraine preventive treatments were unsuccessful, not tolerated, or contraindicated. Erenumab, fremanezumab, and galcanezumab were not evaluated in patients with $\mathrm{CM}$ refractory to current available medical treatments. However, due to the poor quality of life of patients with refractory $\mathrm{CM}$ it is reasonable to treat them in daily clinical practice with erenumab, fremanezumab, or galcanezumab.

\section{Medication overuse}

Some of the available evidence indicates that CM patients with medication overuse (MO) should be 
withdrawn before offering preventive medications, but precise indications on detoxification strategies are not provided [55]. RCTs on erenumab, fremanezumab and galcanezumab enrolled a consistent proportion of patients with $\mathrm{MO}[26,28,31]$. In all those RCTs patients with $\mathrm{MO}$ were not treated with detoxification strategies before the administration of CGRP(r) MoAbs. Preliminary data of a post-hoc analysis of the phase III HALO-CM trial [56] indicates that fremanezumab at monthly and quarterly dosing are both efficacious in the reduction of MMD in patients with $\mathrm{CM}$ and $\mathrm{MO}$ [56]. Moreover, among CM patients with baseline $\mathrm{MO}$, the proportion of those reporting no MO during the 12 -week treatment period was significantly higher in the quarterly (55\%) and monthly (61\%) fremanezumab group compared with placebo (46\%). Among those patients who reverted to no MO, the monthly average number of days with acute headache medication use significantly decreased with both quarterly and monthly fremanezumab compared with placebo [56]. Therefore, it might be reasonable to offer treatment with CGRP(r) MoAbs to patients with $\mathrm{CM}$ and $\mathrm{MO}$. In order to have a clearer picture of the migraine impact and on the effect of CGRP on migraine relief, it would be preferable to detoxify first with later or concomitant initiation of CGRP(r) MoAbs. Anyway, treatment with CGRP(r) MoAbs can be initiated even if detoxification is not feasible, contributing itself to the reduction of the number of acute headache medication.

\section{Association with other concomitant migraine preventive treatments}

Available evidence suggests that CGRP(r) MoAbs are effective and well-tolerated even when added to other concomitant migraine preventive treatments [57, 58]. These results plausibly outline the absence of drug-drug interactions, specificity and safety of CGRP(r) MoAbs.

The add-on strategies might be especially useful in patients who experience an insufficient response to a single preventive treatment. Indeed, in patients who achieved a meaningful efficacy with a preventive medication, but migraine attack frequency or severity still produces too much disability, it would be reasonable not to stop the ongoing treatment and consider adding CGRP(r) MoAbs. Also patients that need to discontinue the preventive treatment due to the lack of efficacy or side effect would benefit from the add-on strategy with CGRP(r) MoAbs during the slow tapering phase, in order to avoid possible rebound effects.

Data from fremanezumab suggest that CM patients with concomitant use of preventive medication would benefit more from the monthly than from the quarterly dose regimen; indeed, compared with placebo, fremanezumab monthly but not fremanezumab quarterly determined a significant reduction of the mean number of monthly headache days of at least moderate severity from baseline to week 12 [58]. Nevertheless, further evidence is needed in order to elucidate the appropriate management of CGRP(r) MoAbs treatment in add-on. It is worthy to consider that all RCTs, in order to avoid confounders, did not include among the allowed concomitant preventive treatments, the use of onabotulinumtoxinA for migraine or for any medical or cosmetic reasons requiring injections in the head, face, or neck. Therefore, at the moment, the association of onabotulinumtoxinA with CGRP(r) MoAbs is not supported by evidence. As the mechanism of action of onabotulinumtoxinA is not entirely clear, it cannot be speculated whether the mechanisms of action may be synergic. From the theoretical point of view, no interaction leading to adverse events can be expected, and adding onabotulinumtoxinA and CGRP(r) MoAbs may represent a treatment strategy for the difficult-to-treat patients.

\section{Evaluation and management of response to treatment}

Assessment of response to treatment

The response to CGRP(r) MoAbs, as for all other preventive treatments, should be regularly monitored with pre-established time intervals in order to determine whether meaningful change is occurred and guide the decision-making process. Scheduled follow-up visits every 3 months for patients receiving monthly dose and every 6 months for those on quarterly dose should be considered. The assessment of efficacy and tolerability of preventive treatment should be based on the patients' subjective response and by physicians' expert opinion guided by outcome metrics. Evidence of treatment benefits would be driven by the reduction in MMD, by the reduction of acute migraine-specific medication use, and by the subjective improvement of function compared with the pretreatment period. To monitor those outcomes, the use of a headache diary is reasonable. Validated scale, including the 6-item Headache Impact Test (HIT-6) [59], the Migraine Disability Assessment Score (MIDAS) [60], the Migraine Physical Function Impact Diary (MPFID) [61], the Migraine-Specific Quality of Life Questionnaire (MSQ) [62], and the Patient Global Impression of Severity of Illness (PGI-S) [63] are especially useful for obtaining an objective measure of change in disability in everyday activity, physical impairment, perception of severity of illness, and quality of life in migraine patients.

\section{Management of non-responder patients}

In RCTs on CGRP(r) MoAbs, patients were considered responders if achieving at least $50 \%$ reduction in MMDs $[18,24-31]$. However, in clinical practice the classification of patients as responders or non-responders is less strict. 
In fact, patients may give value not only to the reduction in the MMDs, but also to improvement in pain severity, duration of attacks, response to acute treatments and associated symptoms. In patients with CM, even a $30 \%$ reduction in MMD might be clinical relevant, especially if it is accompanied by improvement in pain intensity, in quality of life, or of the number of headache-free days. In clinical practice, the overall satisfaction of patients is a key parameter to assess treatment benefit.

A proper timing of clinical assessment is also important to assess possible treatment failure. The available RCTs reported that CGRP(r) MoAbs have a rapid onset of efficacy over few days or weeks; however, they also reported that the proportion of patients with at least $50 \%$ MMD reduction progressively increased from week 4 to week 8 and 12 [21, 25, 29-32, 64, 65]. A relevant proportion of patients who were not responding during the first month of treatment, started having a response with prolonged treatment [66]. In the galcanezumab RCTs on EM, at month $6,62 \%$ of patients who were non-responders after the first month achieved at least $50 \%$ reduction in MMD and 20\% achieved at least $75 \%$ reduction [66]. In patients with $\mathrm{CM}$, with continuation of galcanezumab treatment, at month-3, 38\% of patients who were non-responders after the first month achieved at least 30\% reduction in MMDs and 13\% achieved at least 50\% reduction [66]. In particular, the opportunity of a clinical relevant response, defined in this post hoc analysis as at least 30\% reduction in MMD in patients with $\mathrm{CM}$ and as at least $50 \%$ reduction in MMD in patients with EM, was greatest in patients who, during the initial 2 months of treatment, had EM with limited or modest (from at least 10\% to less than 50\% reduction in MMD) early improvement and for those who had $\mathrm{CM}$ and modest early improvement (from at least $30 \%$ to $<50 \%$ reduction in MMD) [66]. According to those post hoc data, patients who do not achieve a clinically meaningful response within the first month of treatment should not discontinue the drug. Patients that after 12-24 weeks of treatment do not have a clinical meaningful response could be considered for discontinuing treatment with CGRP(r) MoAbs.

Shift from one CGRP(r) MoAbs to another might be attempted in non-responders, even if there are no available data supporting this option. Eptinezumab, fremanezumab and galcanezumab have a similar binding action to CGRP, while erenumab targets CGRP receptor. However, since peptides other than CGRP could bind to the CGRP receptor, and CGRP could bind and activate also non- CGRP receptors, it could be interesting to speculate on the possible clinical differences between blocking the peptide or its receptor [41]. Nevertheless, currently there is no evidence suggesting that patients non-responder to MoAbs targeting the peptide would respond to the MoAb targeting the receptor or vice versa.

It has been recently recognized that CGRP receptors can trigger signalling pathways not only when activated on the cell surface, but also when internalized within endosome [67]. Since the receptors within cellular compartments could be inaccessible to current CGRP(r) MoAbs, further studies should clarify the potential influence of these new drugs on CGRP receptor signalling, trafficking, and expression, and the possible consequence of CGRP receptor regulation on the effectiveness of CGRP(r) MoAbs.

\section{Management of responder patients over time}

Data form the open-label extension of the available RCTs showed that response to treatment with CGRP(r) MoAbs is maintained over the whole 9-12 month period; the effectiveness was paralleled by a well tolerated safety profile as shown by the low incidence of adverse events [68-72]. So, at the moment there are not concerns about loss of efficacy or need of dose augmentation with prolonged use.

Up to now, with the available oral drugs or onabotulinumtoxinA, EM is mostly managed with 3-12 months of duration treatment whereas $\mathrm{CM}$ is mostly managed even with short- or long-term treatment. In the absence of clear evidence, it would be anyhow reasonable to stop the treatment with CGRP(r) MoAbs in patients who achieve, after at least 3-6 months of treatment, a stable reduction of migraine attacks to less than 4 days per month. After stopping CGRP(r) MoAbs, patients should be regularly re-evaluated to verify the persistency of low frequency EM. In patients who have migraine improvement but continue to experience more than 5 days per month with migraine it is reasonable to continue treatment.

Data on galcanezumab indicated that $50 \%$ of patients who were sustained responders during the 6-month treatment period, tend to have first loss of response within 4 months from treatment withdrawal [73]. Migraine, as other chronic diseases, may require long-term or even life-long treatment which is now rarely applied because of the poor tolerability of currently available migraine preventive drugs. A short-term, cyclical treatment, may be reasonable in subjects with a relatively low frequency of attacks but may not represent the best option in patients with high-frequency EM or CM.

\section{Adverse events}

Across all available RCTs, CGRP(r) MoAbs showed that most of the treatment-emergent adverse events were mild to moderate in severity [18, 24-32]. Adverse events leading to discontinuation were infrequent (from $\leq 1 \%$ to $4 \%$ ) in clinical trials. The most common reported adverse event was the occurrence of injection site reactions 
including pain, induration, and erythema with the use of subcutaneous formulations [24-32]. Constipation and reduced tolerance to fatigue represent reasons that may lead to discontinuation of treatment in some patients. Other adverse events reported in $\geq 2 \%$ of patients were upper respiratory tract infection, influenza, nausea, sinusitis, nasopharyngitis, arthralgia, pruritis, back pain, muscle spasm, abdominal pain, urinary tract infection, and dizziness [24-32]. No evidence of any hepatotoxicity of CGRP(R) MoAbs was reported.

Being highly specific for CGRP or its receptor, CGRP(r) MoAbs have minimal interaction with the immune system and no immunomodulatory effect [74]. CGRP(r) MoAbs are engineered to have sequences that closely resemble (humanized MoAbs) or are identical (human MoAbs) to human immunoglobulin sequences. For this reason, the risk of immunogenicity is considered low. However, antidrug antibodies can be produced and neutralizing antibodies might interfere with the effectiveness of the drugs. Data from RCTs have shown that the occurrence of neutralizing antibodies is an infrequent event (from 0 to 3.1\%) [24-26, 29-31]. No specific association between the presence of antidrug antibodies and treatment safety and efficacy has been reported. Anyhow, the clinical consequence of neutralizing and binding antibodies should be further elucidated by studies with a long-term follow-up in order to evaluate the possible association with safety concern, and with loss of efficacy and alteration in half-life of CGRP(r) MoAbs. At the moment no evidence supports the need to test antidrug antibodies in clinical practice.

CGRP is involved in physiological mechanisms in the whole body, including the maintenance of cardio- and cerebrovascular homeostasis $[40,41]$, the facilitation of wound healing [75], and the modulation of gastrointestinal motility [76]. Nevertheless, only little evidence on the safety of long-term blockade of the entire CGRP pathway is currently available. In particular, it is crucial the comprehension of the effect of long-term CGRP(r) MoAbs on the vascular system. Indeed, CGRP acts as a vasodilatory safeguard mechanism during cerebral and cardiac ischemia [77], prevents vasospasm and subsequent ischemia after subarachnoid hemorrhage [78], reduces the generation of hypertension [79], and prevents against heart failure via chronotropic and inotropic effects [80]. It is also to be considered that migraine, in particular migraine with aura, is associated with an increased risk of cardio- and cerebrovascular diseases; a link that appears to be mediated by specific interactions among vascular risk factors and genetic, environmental, personality and psychological factors [81-84]. Thus, it is compelling to assess whether the long-term use of CGRP(r) MoAbs determine or facilitate vascular pathologies or if other protective mechanisms come into play in maintaining vascular homeostasis.
Recent evidence revealed that CGRP enhances neovascularization and lymphangiogenesis under pathological conditions [85]. Consequently, CGRP(r) MoAbs may reduce angiogenesis and enhance lymphedema, and may have a deleterious effect on the healing of gastric ulcer and skin wound, but concurrently. On the other hand, they may inhibit tumor-associated angiogenesis providing a therapeutic strategy for cancer treatment [85].

\section{Conclusions}

CGRP(r) MoAbs are efficacious and safe treatments for migraine prevention. Becoming familiar with the practical aspects of CGRP(r) MoAb treatment is important, as their employment in clinical practice will rapidly increase. Ease of use and lack of relevant side effects represent their main strengths making them the more appealing among the migraine preventive treatments. However, because their high costs they cannot represent the first line treatment for migraine prevention but should be reserved for patients who cannot be managed with the oral treatments or with onabotulinumtoxinA. Further large-scale studies are needed to collect long-term follow-up data in order to establish safety with the long-term use, to define and manage treatment response and non-response, and to assess treatment response in patients that were excluded from the available trials, including drug resistant patients and those with $\mathrm{CM}$ concurrently treated with onabotulinumtoxinA.

\section{Abbreviations}

CGRP(r): Calcitonin gene-related peptide (receptor); CM: Chronic migraine; EM: Episodic migraine; MMD: Monthly migraine day; MO: Medication overuse; MoAbs: Monoclonal antibodies; RCT: Randomized clinical trial

\section{Acknowledgements}

Not applicable.

\section{Funding}

The APCs (article processing charges) for the articles in this thematic series The Changing faces of migraine' were made possible through independent educational sponsorship by Eli Lilly. Eli Lilly provided the funds through an educational grant which included enduring materials within the context of a symposium at the 12th European Headache Federation Congress in September 2018, chaired by Paolo Martelletti. This grant was provided to Springer Healthcare IME who organized the symposium and all of the enduring materials. Three of the articles in this thematic series were developed from content presented at the symposium. Eli Lilly were not involved in the planning of the thematic series, the selection process for topics, nor in any peer review or decision-making processes.

The articles have undergone the journal's standard peer review process overseen by the Editor-in-Chief. For articles where the Editor-in-Chief is an author, the peer review process was overseen by one of the other Editors responsible for this thematic series.

\section{Availability of data and materials}

No additional data available.

\section{Authors' contributions}

$C T$ and SS were responsible for interpreting data and for writing the work; $\mathrm{RO}$ and FP were responsible for revising the draft critically for important intellectual content. All authors read and approved the final manuscript.

Ethics approval

Not applicable. 


\section{Consent for publication}

Not applicable.

\section{Competing interests}

SS received honoraria for participating in advisory boards and for speaking at conferences from Allergan, Eli Lilly, Novartis, TEVA.

\section{Publisher's Note}

Springer Nature remains neutral with regard to jurisdictional claims in published maps and institutional affiliations.

\section{Received: 21 February 2019 Accepted: 18 April 2019}

\section{Published online: 06 May 2019}

\section{References}

1. GBD 2015 Disease and injury incidence and prevalence collaborators (2016) Global, regional, and national incidence, prevalence, and years lived with disability for 310 diseases and injuries, 1990-2015: a systematic analysis for the Global Burden of Disease Study 2015. Lancet 388:1545-1602

2. Stovner $L$, Andree C (2010) Prevalence of headache in Europe: a review for the Eurolight project. J Headache Pain. 11:289-299

3. Victor T, Hu X, Campbell J, Buse D, Lipton R (2010) Migraine prevalence by age and sex in the United States: a life-span study. Cephalalgia. 30:1065-1072

4. Bigal ME, Serrano D, Reed M, Lipton RB (2008) Chronic migraine in the population - burden, diagnosis, and satisfaction with treatment. Neurology. 71:559-566

5. Amara SG, Jonas V, Rosenfeld MG, Ong ES, Evans RM (1982) Alternative RNA processing in calcitonin gene expression generates mRNAs encoding different polypeptide products. Nature. 15(298):240-244

6. Rosenfeld MG, Mermod JJ, Amara SG, Swanson LW, Sawchenko PE, Rivier J, Vale WW, Evans RM (1983) Production of a novel neuropeptide encoded by the calcitonin gene via tissue-specific RNA processing. Nature. 304:129-135

7. Goadsby PJ (2016) Bench to bedside advances in the 21st century for primary headache disorders: migraine treatments for migraine patients. Brain. 139:2571-2577

8. Ho TW, Edvinsson L, Goadsby PJ (2010) CGRP and its receptors provide new insights into migraine pathophysiology. Nat Rev Neurol 6:573-582

9. Goadsby PJ, Edvinsson L, Ekman R (1990) Vasoactive peptide release in the extracerebral circulation of humans during migraine headache. Ann Neurol 28:183-187

10. Goadsby PJ, Edvinsson $L$ (1993) The trigeminovascular system and migraine: studies characterizing cerebrovascular and neuropeptide changes seen in humans and cats. Ann Neurol 33:48-56

11. Juhasz G, Zsombok T, Modos EA, Olajos S, Jakab B, Nemeth J, Szolcsanyi J, Vitrai J, Bagdy G (2003) NO-induced migraine attack: strong increase in plasma calcitonin gene-related peptide (CGRP) concentration and negative correlation with platelet serotonin release. Pain. 106:461-470

12. Lassen LH, Haderslev PA, Jacobsen VB, Iversen HK, Sperling B, Olesen J (2002) CGRP may play a causative role in migraine. Cephalalgia. 22:54-61

13. Petersen KA, Lassen LH, Birk S, Lesko L, Olesen J (2005) BIBN4096BS antagonizes human alpha-calcitonin gene related peptide-induced headache and extracerebral artery dilatation. Clin Pharmacol Ther 77:202-213

14. Edvinsson L, Warfvinge K (2017) Recognizing the role of CGRP and CGRP receptors in migraine and its treatment. Cephalalgia 39:366-73

15. Olesen J, Burstein R, Ashina M, Tfelt-Hansen P (2009) Origin of pain in migraine: evidence for peripheral sensitisation. Lancet Neurol 8:679-690

16. González-Hernández A, Marichal-Cancino BA, MaassenVanDenBrink A, Villalón CM (2018) Side effects associated with current and prospective antimigraine pharmacotherapies. Expert Opin Drug Metab Toxicol 14:25-41

17. Edvinsson $L$ (2017) The trigeminovascular pathway: role of CGRP and CGRP receptors in migraine. Headache. 57:47-55

18. Dodick DW, Goadsby PJ, Silberstein SD, Lipton RB, Olesen J, Ashina M, Wilks K, Kudrow D, Kroll R, Kohrman B, Bargar R, Hirman J, Smith J (2014) ALD403 study investigators. Safety and efficacy of ALD403, an antibody to calcitonin gene-related peptide, for the prevention of frequent episodic migraine: a randomised, double-blind, placebo-controlled, exploratory phase 2 trial. Lancet Neurol 13:1100-1107

19. Saper J, Lipton R, Kudrow D, Hirman J, Dodick D, Silberstein S, Chakhava G, Smith J (2018) Primary results of PROMISE-1 (PRrevention of migraine via intravenous eptinezumab safety and efficacy-1) trial: a phase 3, randomized, double-blind, placebo-controlled study to evaluate the efficacy and safety of eptinezumab for prevention of frequent episodic migraines. Neurology. 90(15 Supplement):S20.001

20. Silberstein S, McAllister P, Berman G, Cady R, Smith J, Biondi D, Hirman J (2018) Eptinezumab reduced migraine frequency, duration, and pain intensity through week 24: results from the phase 3 PROMISE-1 trial. Neurology. 90(15 Supplement):4091

21. Smith T, Biondi D, Berman G, Freeman M, Hirman J, Kassel E (2018) Eptinezumab achieved meaningful reductions in migraine activity within 24 hours that were sustained through week 12: results from PROMISE-1 (prevention of migraine via intravenous eptinezumab safety and Efficacy-1) phase 3 trial. Neurology. 90(15 Supplement):4.092

22. Lipton RB, Saper J, Ashina M, Biondi D, Bhattacharya S, Hirman J, Schaeffler B, Cady R (2018) A phase 3 study to evaluate eptinezumab for the preventive treatment of chronic migraine: results of the PROMISE-2 (PRevention of migraine via intravenous Eptinezumab safety and Efficacy-2) trial. Headache. 50(S2):PF02

23. Winner PK, Goadsby P, Nagy A, Brandes J, Biondi D, Bhattacharya S, Cady R, Hirman J, Kassel E (2018) Eptinezumab reduced migraine activity and achieved high migraine responder rates over weeks 1-12: results from the phase 3 PROMISE-2 trial in chronic migraine. Headache. 50(S2):IOR03

24. Goadsby PJ, Reuter U, Hallström Y, Broessner G, Bonner JH, Zhang F, Sapra S, Picard H, Mikol DD, Lenz RA (2017) A controlled trial of erenumab for episodic migraine. N Engl J Med 377:2123-2132

25. Dodick DW, Ashina M, Brandes JL, Kudrow D, Lanteri-Minet M, Osipova V, Palmer K, Picard H, Mikol DD, Lenz RA (2018) ARISE: a phase 3 randomized trial of erenumab for episodic migraine. Cephalalgia. 38:1026-1037

26. Tepper S, Ashina M, Reuter U, Brandes JL, Doležil D, Silberstein S, Winner P, Leonardi D, Mikol D, Lenz R (2017) Safety and efficacy of erenumab for preventive treatment of chronic migraine: a randomised, double-blind, placebo-controlled phase 2 trial. Lancet Neurol 16:425-434

27. Dodick DW, Silberstein SD, Bigal ME, Yeung PP, Goadsby PJ, Blankenbiller T, Grozinski-Wolff M, Yang R, Ma Y, Aycardi E (2018) Effect of fremanezumab compared with placebo for prevention of episodic migraine: a randomized clinical trial. JAMA. 319:1999-2008

28. Silberstein SD, Dodick DW, Bigal ME, Yeung PP, Goadsby PJ, Blankenbiller T, Grozinski-Wolff M, Yang R, Ma Y, Aycardi E (2017) Fremanezumab for the preventive treatment of chronic migraine. N Engl J Med 377:2113-2122

29. Stauffer VL, Dodick DW, Zhang Q, Carter JN, Ailani J, Conley RR (2018) Evaluation of galcanezumab for the prevention of episodic migraine: the EVOLVE-1 randomized clinical trial. JAMA Neurol 75:1080-1088

30. Skljarevski V, Matharu M, Millen BA, Ossipov MH, Kim BK, Yang JY (2018) Efficacy and safety of galcanezumab for the prevention of episodic migraine: results of the EVOLVE-2 phase 3 randomized controlled clinical trial. Cephalalgia. 38:1442-1454

31. Detke HC, Goadsby PJ, Wang S, Friedman DI, Selzler KJ, Aurora SK (2018) Galcanezumab in chronic migraine: the randomized, double-blind, placebocontrolled REGAIN study. Neurology. 91:e2211-e2221

32. Skljarevski V, Oakes TM, Zhang Q, Ferguson MB, Martinez J, Camporeale A, Johnson KW, Shan Q, Carter J, Schacht A, Goadsby PJ, Dodick DW (2018) Effect of different doses of galcanezumab vs placebo for episodic migraine prevention: a randomized clinical trial. JAMA Neurol. 75:187-193

33. Silberstein SD, Rapoport AM, Loupe PS, Aycardi E, McDonald M, Yang R, Bigal ME (2019) The effect of beginning treatment with fremanezumab on headache and associated symptoms in the randomized phase 2 study of high frequency episodic migraine: post-hoc analyses on the first 3 weeks of treatment. Headache. 59:383-393.

34. Brandes JL, Yeung PP, Aycardi E, Yang R, Ma Y, Cohen JM (2018) The impact of fremanezumab on symptoms associated with migraine in patients with episodic migraine. J Headache Pain. 19(Suppl 1):P3

35. McAllister P, Yeung PP, Aycardi E, Yang R, Ma Y, Cohen JM (2018) The impact of fremanezumab on symptoms associated with migraine in patients with chronic migraine. J Headache Pain. 19(Suppl 1):P4

36. Day KA, Ament M, Stauffer VL, Skljarevski V, Zhang Q, Pearlman EM, Aurora SK (2018) Effect of galcanezumab on severity and symptoms of migraine in phase 3 trials in patients with episodic or chronic migraine. J Headache Pain. 19(Suppl 1):O31

37. Hadjikhani N, Sanchez Del Rio M, Wu O, Schwartz D, Bakker D, Fischl B, Kwong KK, Cutrer FM, Rosen BR, Tootell RB, Sorensen AG, Moskowitz MA (2001) Mechanisms of migraine aura revealed by functional MRI in human visual cortex. Proc Natl Acad Sci U S A 98: 4687-4692 
38. Headache Classification Committee of the International Headache Society (IHS) (2013) The International Classification of Headache Disorders, 3rd edition (beta version). Cephalalgia 33:629-808

39. Sacco S, Bendtsen L, Ashina M, Reuter U, Terwindt G, Mitsikostas DD, Martelletti P (2019) European headache federation guideline on the use of monoclonal. J Headache Pain. 20:6

40. Uddman R, Edvinsson L, Ekblad E, Håkanson R, Sundler F (1986) Calcitonin gene-related peptide (CGRP): perivascular distribution and vasodilatory effects. Regul Pept 15:1-23

41. MaassenVanDenBrink A, Meijer J, Villalón CM, Ferrari MD (2016) Wiping Out CGRP: Potential cardiovascular risks. Trends Pharmacol Sci 37(9):779-788

42. Ohlsson L, Kronvall E, Stratton J, Edvinsson L (2018) Fremanezumab blocks CGRP induced dilatation in human cerebral middle meningeal and abdominal arteries. J Headache Pain. 19:66

43. Depre C, Antalik L, Starling A, Koren M, Eisele O, Lenz RA, Mikol DD (2018) A randomized, double-blind, placebo-controlled study to evaluate the effect of erenumab on exercise time during a treadmill test in patients with stable angina. Headache. 58:715-723

44. Tepper S, Pascual J, Reuter U, Picard H, Hong F, Trotman ML, Xue F, Mikol DD, Klatt J (2018) Analysis of blood presuure following erenumab treatment. Analysis of blood pressure following short-term and long-term treatment with erenumab. Neurology 90(15 Supplement):4.103

45. Tepper S, Broessner G, Buse G, Schwedt T, Strauss E, Zhang F, Picard H, Mikol D (2018) Efficacy of erenumab for the treatment of patients with episodic migraine with depression and/or anxiety. Neurology. 90(15 Supplement):4.105

46. Cohen JM, Yeung PP, Aycardi E, Bigal ME, Yang R, Bibeau K, Galic M, Seminerio MJ, Lipton RB, Buse DC (2018) Efficacy of fremanezumab in patients with chronic migraine and comorbid moderate to moderately severe depression. Neurology. 90(15 Supplement):4.097

47. Ryman JT, Meibohm B (2017) Pharmacokinetics of monoclonal antibodies. CPT Pharmacometrics Syst Pharmacol 6:576-588

48. Goadsby PJ, Paemeleire K, Broessner G, Brandes J, Klatt J, Zhang F, Picard H, Mikol D (2017) Efficacy of erenumab in subjects with episodic migraine with prior preventive treatment failure(s). Cephalalgia. 37(Suppl 1):13

49. Ashina M, Tepper S, Brandes JL, Reuter U, Boudreau G, Dolezil D, Cheng S, Zhang F, Lenz R, Klatt J, Mikol DD (2018) Efficacy and safety of erenumab (AMG334) in chronic migraine patients with prior preventive treatment failure: a subgroup analysis of a randomized, double-blind, placebocontrolled study. Cephalalgia. 38:1611-1621

50. Winner PK, Singh RBH, Cohen JM, Yang R, Yeung PP, Campos VR (2018) Impact of fremanezumab on response rates, acute medication use, and disability in patients with episodic migraine who have failed at least one prior migraine preventive medication. J Headache Pain. 19(Suppl 1):O38

51. Silberstein SD, Ailani J, Cohen JM, Yang R, Yeung PP, Campos VR (2018) Impact of fremanezumab on response rates, migraine days, and acute medication use in patients with chronic migraine who have failed at least one prior migraine preventive medication. J Headache Pain. 19(Suppl 1):039

52. Dell'Agnello G, Tockhorn-Heidenreich A, Zhang Q, Ruff DD, Pearlman EM, Aurora SK, Ford JH (2018) Efficacy of galcanezumab in patients who failed prior preventive treatments for migraine: results from EVOLVE-1, EVOLVE-2 and REGAIN studies. J Headache Pain. 19(Suppl 1):019

53. Reuter U, Goadsby PJ, Lanteri-Minet M, Wen S, Hours-Zesiger P, Ferrari MD, Klatt J (2018) Efficacy and tolerability of erenumab in patients with episodic migraine in whom two-to-four previous preventive treatments were unsuccessful: a randomised, double-blind, placebo-controlled, phase $3 \mathrm{~b}$ study. Lancet. 392:2280-2287

54. Ailani J, Pearlman E, Zhang Q, Nagy AJ, Schuh K, Aurora SK (2018) Positive response to galcanezumab following treatment failure to onabotulinumtoxinA in patients with migraine. Poster PS106LB presented at the American Headache Society (AHS), San Francisco

55. de Goffau MJ, Klaver ARE, Willemsen MG, Bindels PJE, Verhagen AP (2017) The effectiveness of treatments for patients with medication overuse headache: a systematic review and meta-analysis. J Pain 18:615-627

56. Silberstein SD, Ashina S, Katsarava Z, Bibeau K, Seminerio MJ, Harlow DE, Cohen JM (2018) The impact of fremanezumab on medication overuse in patients with chronic migraine. J Headache Pain. 19(Suppl 1):12

57. Cohen JM, Dodick DW, Yang R, Newman LC, Li T, Aycardi E, Bigal ME (2017) Fremanezumab as add-on treatment for patients treated with other migraine preventive medicines. Headache. 57:1375-1384

58. Goadsby PJ, Dodick DW, Silberstein SD, Yeung PP, Blankenbiller T, Ning X, Yang R, Ma Y, Aycardi E, Bigal ME (2018) Efficacy of fremanezumab in patients with chronic migraine with or without concomitant use of preventive medication. J Headache Pain. 19(Suppl 1):8

59. Rendas-Baum R, Yang M, Varon SF, Bloudek LM, DeGryse RE, Kosinski M (2014) Validation of the headache impact test (HIT-6) in patients with chronic migraine. Health Qual Life Outcomes 12:117

60. Lipton RB, Stewart WF, Sawyer J, Edmeads JG (2001) Clinical utility of an instrument assessing migraine disability: the migraine disability assessment (MIDAS) questionnaire. Headache. 41(9):854-861

61. Kawata AK, Hsieh R, Bender R, Shaffer S, Revicki DA, Bayliss M, Buse DC, Desai P, Sapra S, Ortmeier B, Hareendran A (2017) Psychometric evaluation of a novel instrument assessing the impact of migraine on physical functioning: the migraine physical function impact diary. Headache. 57:1385-1398

62. Cole JC, Lin P, Rupnow MFT (2007) Validation of the migraine-specific quality of life questionnaire version 2.1 (MSQ v. 2.1) for patients undergoing prophylactic migraine treatment. Qual Life Res 16:1231-1237

63. Guy W (1976) ECDEU assessment manual for psychopharmacology, revised. National Institute of Mental Health, Psychopharmacology Research Branch, Rockville, pp 217-222

64. Schwedt T, Reuter U, Tepper S, Ashina M, Kudrow D, Broessner G, Boudreau GP, McAllister P, Vu T, Zhang F, Cheng S, Picard H, Wen S, Kahn J, Klatt J, Mikol D (2018) Early onset of efficacy with erenumab in patients with episodic and chronic migraine. J Headache Pain. 19:92

65. Aurora SK, Detke HC, Millen BA, Hundemer HP (2018) Rapid onset of effect of galcanezumab for the prevention of episodic migraine: post-hoc analyses of two phase 3 studies. J Headache Pain. 19(Suppl 1):132

66. Nichols R, Doty E, Sacco S, Ruff D, Pearlman E, Aurora SK (2019) Analysis of initial nonresponders to galcanezumab in patients with episodic or chronic migraine: results from the EVOLVE-1, EVOLVE-2, and REGAIN randomized, double-blind, placebo-controlled studies. Headache. 59:192-204

67. Gingell JJ, Hendrikse ER, Hay DL (2019) New insights into the regulation of CGRP-family receptors. Trends Pharmacol Sci 40:71-83

68. Detke HC, Li LQ, Wang S, Aurora SK (2018) One-year treatment with galcanezumab in patients with chronic migraine: results from the openlabel phase of the REGAIN study. Cephalalgia. 38(1S):149

69. Camporeale A, Kudrow D, Sides R, Wang S, Van Dycke A, Selzler KJ, Stauffer VL (2018) A phase 3, long-term, open-label safety study of Galcanezumab in patients with migraine. BMC Neurol 18:188

70. Tepper S, Ashina M, Reuter U, Brandes JL, Doležil D, Silberstein S, Winner P, Zhang F, Cheng S, Mikol D (2018) Assessment of the long-term safety and efficacy of erenumab during open-label treatment of subjects with chronic migraine. J Headache Pain 19(Suppl 1):24

71. Ashina M, Dodick D, Goadsby PJ, Reuter U, Silberstein S, Zhang F, Gage JR, Cheng S, Mikol DD, Lenz RA (2017) Erenumab (AMG 334) in episodic migraine: interim analysis of an ongoing open-label study. Neurology. 89:1237-1243

72. Brandes JL, Yeung PP, Cohen JM, Gandhi SK, Fitzgerald T, Yang R, Ma Y, Aycardi $E$ (2018) Long-term impact of fremanezumab on response rates, acute headache medication use, and disability in patients with episodic migraine: interim results of a 1-year study. J Headache Pain 19(Suppl 1):5

73. Z Zhang Q, Morrow PA, Stauffer VL, Skljarevski V, Pearlman EM, Aurora SK (2018) Effect of galcanezumab following double-blind treatment in patients with migraine: results from EVOLVE-1 and EVOLVE-2. J Headache Pain 19(Suppl 1):19

74. Silberstein S, Lenz R, Xu C (2015) Therapeutic monoclonal antibodies: what headache specialists need to know. Headache. 55:1171-1182

75. Khalil Z, Helme R (1996) Sensory peptides as neuromodulators of wound healing in aged rats. J Gerontol A Biol Sci Med Sci 51:B354-B361

76. Sun YD, Benishin CG (1991) Effects of calcitonin gene-related peptide on longitudinal muscle and myenteric plexus of Guinea pig ileum. J Pharmacol Exp Ther 259:947-952

77. Russell FA, King R, Smillie SJ, Kodji X, Brain SD (2014) Calcitonin gene-related peptide: physiology and pathophysiology. Physiol Rev 94:1099-1142

78. Juul R, Edvinsson L, Gisvold SE, Ekman R, Brubakk AO, Fredriksen TA (1990) Calcitonin gene-related peptide-LI in subarachnoid haemorrhage in man. Signs of activation of the trigemino-cerebrovascular system? $\mathrm{Br}$ J Neurosurg 4:171-179

79. Smillie SJ, King R, Kodji X, Outzen E, Pozsgai G, Fernandes E, Marshall N, de Winter P, Heads RJ, Dessapt-Baradez C, Gnudi L, Sams A, Shah AM, Siow RC, Brain SD (2014) An ongoing role of a-calcitonin gene-related peptide as part of a protective network against hypertension, vascular hypertrophy, and oxidative stress. Hypertension. 63:1056-1062 
80. Saetrum Opgaard O, Hasbak P, de Vries R, Saxena PR, Edvinsson L (2000) Positive inotropy mediated via CGRP receptors in isolated human myocardial trabeculae. Eur J Pharmacol 397:373-382

81. Sacco S, Cerone D, Carolei A (2008) Comorbid neuropathologies in migraine: an update on cerebrovascular and cardiovascular aspects. J Headache Pain 9:237-248

82. Sacco S, Pistoia F, Degan D, Carolei A (2015) Conventional vascular risk factors: their role in the association between migraine and cardiovascular diseases. Cephalalgia. 35:146-164

83. Sacco S, Kurth T (2014) Migraine and the risk for stroke and cardiovascular disease. Curr Cardiol Rep 16:524

84. Sacco S, Ripa P, Grassi D, Pistoia F, Ornello R, Carolei A, Kurth T (2013) Peripheral vascular dysfunction in migraine: a review. J Headache Pain. 14:80

85. Majima M, Ito Y, Hosono K, Amano H (2019) CGRP/CGRP receptor antibodies: potential adverse effects due to blockade of neovascularization? Trends Pharmacol Sci 40:11-21

Ready to submit your research? Choose BMC and benefit from:

- fast, convenient online submission

- thorough peer review by experienced researchers in your field

- rapid publication on acceptance

- support for research data, including large and complex data types

- gold Open Access which fosters wider collaboration and increased citations

- maximum visibility for your research: over $100 \mathrm{M}$ website views per year

At BMC, research is always in progress.

Learn more biomedcentral.com/submissions 